Classification

Physics Abstracts

$32.80 \mathrm{M}-79.20 \mathrm{~F}$

\title{
The Auger Effect
}

Marie-Geneviève Barthés-Labrousse

CNRS, Centre d'Etudes de Chimie Métallurgique, 15, rue Georges Urbain, 94407 Vitry Cedex, France

(Received April 1; accepted April 16, 1995)

\begin{abstract}
A brief history of the discovery of the Auger effect is given, which is followed by a short description of some of the fields in which this discovery has had a major impact.
\end{abstract}

\section{Introduction}

Pierre Auger was born in Paris in 1899. After his university studies and agregation, he joined, in 1922, the Laboratoire de Chimie Physique, headed by Jean Perrin, to prepare a thesis on the photoelectric effect. A few months later, he published the first description of the phenomenon which now bears his name [1].

However, thirty years passed until the technology sufficiently advanced to allow this major discovery to be put in use by the scientific communauty. The Auger effect has now proved to be particularly fruitful in many fields like fundamental physics (with the study of atoms, molecules, collision processes...) or fundamental and applied surface science.

In this article, we will first give a brief history of the discovery of the Auger effect. This will be followed by a short description of some of the fields in which this discovery has had a major impact.

\section{The Discovery of the Auger Effect}

Following the discovery of the photoelectric effect by Hertz in 1887, X-ray photoelectron spectroscopy developed rapidly. In the early 1920s, the photoelectron spectra of many elements had already been obtained.

At those times, most of the experiments on the photoelectric effect were carried out using magnetic analysis and photographic detection. However, C.T.R. Wilson had proposed in 1912 an experimental setting (the cloud chamber) which allowed a direct observation of the photoelectron trajectories [2]. When a chamber filled with air saturated in water is illuminated with an 
monochromatic X-ray beam, the interaction of a photon $h \nu$ with a gas molecule induces ionisation. The photon energy is transferred to an electron, called photoelectron (or secondary $\beta$ ), which is then ejected with a kinetic energy given by:

$$
E_{\mathrm{k}}=h \nu-E_{1}
$$

where $h \nu$ is the incident photon energy and $E_{1}$ the electron binding energy. This electron can in turn, induce further ionisation of the gas molecules along its trajectory. Because ionised molecules favor condensation of water, the trajectory of the photoelectron can be materialized by the appearance of droplets. The length of the trajectory is then a direct measurement of the kinetic energy of the ejected photoelectron.

In 1922, Pierre Auger starts his research activities by constructing, with his friend Francis Perrin, the first Wilson expansion chamber in France. By changing the experimental conditions and filling the chamber with argon, he notices that photoelectron trajectories contain a tiny group of small droplets at the start of each trajectory. In the early twenties, the only process of desexcitation admitted for an ionised atom is $\mathrm{X}$ - fluorescence, for which the initial core hole is filled with a shallower level electron of binding energy $E_{2}$, the energy balance being taken by emission of a secondary photon $h \nu^{\prime}$ (see Fig. 1):

$$
h \nu^{\prime}=E_{1}-E_{2}
$$

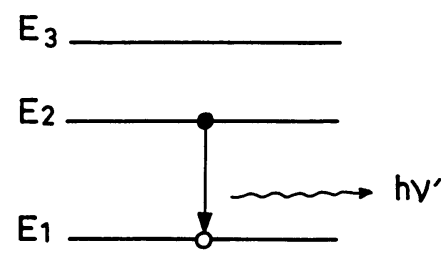

Fig. 1. - Radiative decay (X-ray fluorescence).

The droplets observed by Pierre Auger correspond to the presence of ions which cannot be explained by the fluorescence theory. In fact, dilution of the argon in hydrogen to lengthen the electron tracks allows him to observe that these droplets are new short electron trajectories originating at the same point as that of the photoelectron, as noted in his early paper dated 1923:

"On remarque la présence presque constante d'une trajectoire très ionisante...qui part du même point que le rayon $\beta$ secondaire... Il y a donc émission d'un second électron plus lent." [1]

Moreover, experiments carried out with different gases (chlorine, iodine, nitrogen) and different energies of the incident X-ray beam show that the length of the trajectory, i.e. the energy of the ejected electron (called tertiary $\beta$ ), depends on the nature of the ionised atom but is independent of the incident beam energy:

"Une étude rapide des propriétés de ces rayons $\beta$ tertiaires a conduit aux remarques suivantes: leur vitesse, évaluée par les parcours, dépend de la nature de l'atome dont ils proviennent, et ne varie pas, au moins dans certaines limites, avec la fréquence du rayonnement X primitif." [1]

Pierre Auger initially interpreted this new effect in terms of internal reabsorption of the characteristic (fluorescence) radiation emitted following initial ionisation and proposed that this reabsorption lead to the emission of a second electron:

"Lors du départ du premier électron, sous forme du rayon $\beta$ secondaire, une place est laissée libre dans le système électronique de l'atome excité. La chute d'un électron plus périphérique sur ce niveau 
s'accompagne de l'émission d'un quantum de rayonnement caractéristique. Ce quantum peut être absorbé dans l'atome même, et servir à la production, aux dépends des niveaux périphériques, d'un rayon $\beta$ tertiaire." [1]

Moreover, if the final state has a vacancy in a core-level, the same process can give rise to a third electron (quaternary $\beta$ ):

"La répétition du même processus doit aboutir à la production d'un rayon quaternaire; et je crois en effet avoir observé de tels rayons dans le cas de l'iode." [1]

Such an interpretation in terms of internal conversion process was analogous to the theory proposed by Ellis [3] and Meitner [4] to explain the emission of electrons in radioactive sources ( $\beta$-rays):

"Die primären $\beta$-Strahlen von etwa 59 Proz. $C$ werfen $K$-Elektronen heraus, dadurch wird die $K_{\alpha}$ Strahlung angeregt, die ihrerseits wieder L-, M- oder $N$-Elektronen auslöst, wobei sich der ganze Vorgang von der mit dem Kernzerfall verknüpfen Aussendung des primären $\beta$-Strahls an bis zur Auslösung eines L-, M-oder $N$-Elektrons im selben Atom abspielt. Natürlich mu $\beta$ dabei auch die charakteristische L-, M-, usw.-Serie angeregt werden." [4]

However, Pierre Auger soon realised that emission of the tertiary $\beta$ electrons was not a consequence of the radiative fluorescence effect, but an alternative non-radiative decay process. For this internal radiationless transition, the initial inner-shell vacancy is filled with a shallower level electron as for fluorescence, but, in this case, the energy balance is removed by direct ejection of a third electron (tertiary $\beta$ ) from an outer shell [5]:

'Le processus de sortie sous forme électromagnétique n'est donc pas nécessaire, et l'énergie libérée par le premier retour électronique peut aussi quitter l'atome sous forme corpusculaire...laissant l'atome ionisé deux fois." [5]

The kinetic energy $E_{\mathrm{A}}$ of the ejected electron is then given by

$$
E_{\mathrm{A}}=E_{1}-E_{2}-E_{3}^{*}
$$

where $E_{3}^{*}$ is the binding energy of an electron in the level 3 in the presence of a hole in the level 1. Following the relaxation process, the atom is left in a double-ionised state with vacancies in levels 2 and 3 (see Fig. 2).

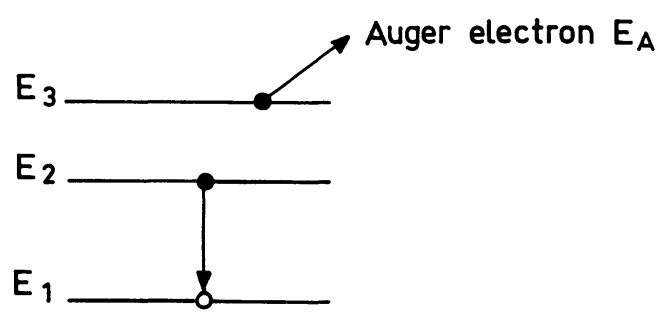

Fig. 2. - Non-radiative decay (Auger process).

Finally, in his $\mathrm{PhD}$ presentation in 1926 [6], Pierre Auger proposed a complete description of what he named "l'effet photoélectrique composé", which soon became the "Auger effect". By that time, he had already observed the most salient features of this new phenomenon. In particular, he noticed that:

i) Auger electrons can be obtained following initial ionisation by incident electrons (either 
photoelectrons or electrons originating from a radioactive source) instead of photons: "Avec les photoélectrons je n'ai pu observer que l'effet dans $L$ : il donne lieu à la production de trajectoires adjacentes identiques aux tertiaires $L$ ou quaternaires de l'effet photoélectrique complexe dans $K$."[6]

ii) for core states with low-binding energies, the more probable decay mechanism following ionisation is through ejection of an electron. This means that it will be the dominant mechanism for light elements. However, it will also be the preponderant mechanism for shallow levels of heavy elements. This observation allowed Pierre Auger to explain the poor fluorescence yield observed by Holweck in some anticathodes when irradiated with soft X-rays [7]:

"D'autre part les niveaux périphériques des atomes lourds se conduisent à peu près de la même manière que les niveaux profonds des corps légers, et leur rendement de fluorescence peut devenir très petit." [6] iii) the Auger electrons are emitted in all directions (isotropic emission):

"La répartition ainsi trouvée indique nettement que l'atome ne garde pas (ou presque pas) de souvenir de la direction des rayons $X$ qui l'ont primitivement ionisé. Il est probable qu'il a eu, pendant sa durée d'existence à l'état excité, le temps de tourner sur lui-même d'une façon plus ou moins compliquée et de ne rien garder de son orientation primitive." [6]

Thus, fourty years after the discovery of atomic radiative processes, the Röntgen Rays, the complementary non radiative processes were discovered by Pierre Auger. And it was soon realised that Auger transitions play an important role in all phenomena subsequent to inner-shell ionisation.

A period of slow but continuous development followed the discovery of Pierre Auger. In 1927, Wentzel laid the foundation of the theory of the Auger effect [8]. In the following years, evidence of the occurence of radiationless transitions was provided in many fields. In X-ray spectroscopy, transitions of the Auger type were shown to influence the width of X-ray emission lines, their intensities and the appearance of satellites lines. For instance, Coster and Kronig [9] found that the abnormally weak intensity of lines arising from an $L_{1}$ initial state of ionisation observed for many elements was due to a special type of Auger process in which the final doubly charged ion has one hole in a shell of the same principal quantum number as that of the original ion. In 1935, Howarth reported the presence of Auger peaks in the secondary-electron spectra of electronbombarded targets [10]. The importance of radiationless transitions in auto-ionisation of helium (auto-ionisation) was recognised in 1936 by Kiang et al. [11]. Radiationless transitions between excited states of molecules can also give rise to predissociation phenomena. Radiationless collisions bearing a close relation to the Auger effect and grouped under the heading of collisions of the second kind were reviewed by Massey and Burhop [12].

However, it is not until the 1960s that technological improvement allowed the activity in the field of Auger spectroscopy to expand rapidly. A symposium dedicated to the Auger effect was held in Paris, March 1989 upon the occasion of Pierre Auger's 90th birthday, during which one could realise the colossal impact of Pierre Auger's major discovery in two fields: fundamental physics and surface science.

\section{The Auger Effect and Fundamental Physics}

Great investments were made in the late sixties and early seventies to understand the physics of the Auger effect. The tremendous utility of the Auger effect as a probing tool to give new insights into the electronic structure and dynamics of atoms has recently been reviewed by Briant in a special issue of "Le Vide, Les Couches Minces" dedicated to Pierre Auger [13]. We will present here only a few examples of the recent surge on research activity in this field. 
3.1 RAdiative Auger Effect. - Decay of an inner-hole state by simultaneous emission of a photon and an electron was first postulated in 1935 by Bloch [14] as an alternative to a dipole forbidden X-ray transition. The principle of this radiative Auger effect is shown in Figure 3. It is a double-electron transition where the filling of an inner-shell vacancy by a less tightly bound electron is accompanied by the simultaneous emission of a photon and excitation of another electron

into a bound or continuum level of the atom. The energy balance is then shared between the Auger electron and the emitted photon:

$$
E_{\mathrm{A}}+h \nu^{\prime}=\left(E_{1}-E_{2}-E_{3}^{*}\right)
$$

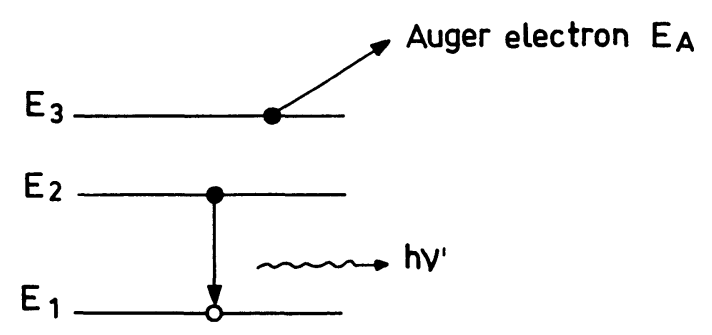

Fig. 3. - Radiative Auger effect.

Consequently, these transitions give rise to continuous spectral distribution. The interest in the radiative Auger effect has been reniewed in the early seventies, with the observation by Aberg and his coworkers [15] of low-energy structures accompanying ordinary X-ray lines. These authors have shown that the radiative Auger effect can be due to the change in the average potential acting on the electrons in the atom when the inner-shell vacancy moves to an outer-shell (shake-off), and to the interaction between single- and double-hole configurations in the final state (configuration interaction).

3.2 Production of Multiply Charged Ions. - The application of the Auger effect in the study of energetic ion-atom collisions has been reniewed by the availability of high-energy accelerators and high-temperature plasmas. In the field of target spectroscopy, neutral atoms are multiply ionised using heavy projectiles in a single collision. However, minimisation of kinematic recoil effects can only be achieved using projectiles of sufficiently high energy. Thus, high-resolution Auger spectroscopy of highly ionised atomic species requires high-energy accelerator facilities. In the field of projectile spectroscopy, specific vacancy states can be produced by using light atoms (e.g. He or $\mathrm{H}_{2}$ ) as targets. These atoms behave like a "needle" and can excite the inner-shell of the heavy projectile without affecting the outer-shell. In recent years, "needle" ionisation has often been used to produce multiply charged ions.

3.3 Electron Capture by Highly Charged Ions; Hollow Atoms. - The study of twoelectron capture by multiply charged ions during slow collisions with few-electron targets affords a direct method of observing electron-electron interaction effects in such collisions. In particular, it has been suggested that the correlated transitions producing autoionizing states involve both energy and angular momentum exchange between the two transferred electrons [16]. 
The interaction of slow highly charged ions with solid surfaces results in several effects: emission of a large number of secondary electrons, possibility of a "Coulomb explosion" of atoms near the point of impact of a non-conductive surface or formation of a "hollow atom" with many electrons in high- $\eta$ quantum states. When highly charged ions approach a metal surface at low velocity, they can resonantly capture a large number of electrons from the conduction band of the metal at rather large distances into high Rydberg levels. This capture takes place in a very short time and leads to a situation where most of the electrons are excited in outermost shells while the inner shells are empty (hollow atoms) [17]. Under continued flow of electrons from the metal to the ion, Auger-cascades down to lower lying levels can develop, which are used as internal clocks.

3.4 Internal Dielectronic Excitation (IDE). - It has recently been shown by Schuch et al. [18] that neutralisation of ions on surfaces could also occur through internal dielectronic excitation (IDE). The principle of such a process is illustrated in Figure 4. When two electrons are captured in high- $\eta$ quantum states, they can interact in such a way that one gives birth to an internal vacancy while the other decay to an internal vacant state.

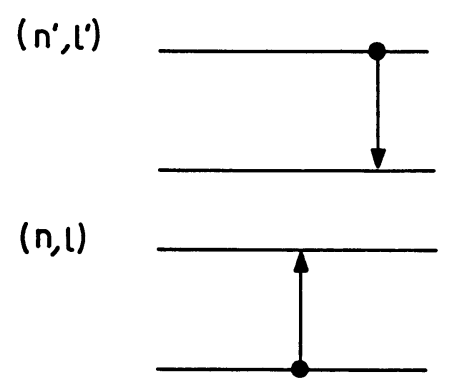

Fig. 4. - Internal dielectronic excitation.

3.5 Dielectronic Recombination (DR). - The dielectronic recombination, as first proposed by Massey and Bates [19], is the simultaneous capture of a free electron and excitation of an ion (see Fig. 5).

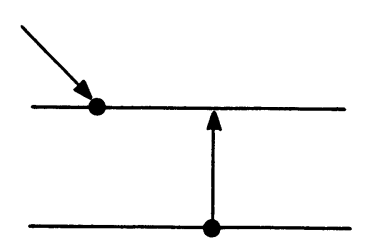

Fig. 5. - Dielectronic recombination.

This process results in a doubly excited state of the atom. When occuring at the energy of the corresponding autoionization electron, it can be considered as the inverse Auger process. This process plays a significant role in controlled fusion devices and it has been extensively studied in 
tokamak plasmas during the last decade, where it provides a reliable measurement of the plasma temperature and density. In principle, cross section measurements of dielectronic recombination provide a direct measurement of the Auger probability. However, such measurements have long been difficult because they required to perform cross beam experiments. An alternative method has been recently proposed [20], which consists in bombarding a target of ions trapped in an electron-beam ion source with the electron beam itself.

\section{The Auger Effect and Surface Science}

For many years surface investigation was limited to clean metal single crystals, studied by low energy electron diffraction (LEED) in glass vacuum systems. Even though it was already recognised in the 1950s that extremely thin layers could have a marked effect on material properties, it is not until the 1960s that instrumental improvement lead to the development of two surface techniques: Auger electron spectroscopy (AES) and X-ray photoelectron spectroscopy (XPS or ESCA).

In 1953, Lander was the first to realise that Auger electrons had great potential for surface chemical analysis [21]:

"In many problems of applied physics and chemistry, the identification of surface composition is at present impossible but of considerable importance. Where the material of interest is present to a depth of several atomic layers and the high vacuum conditions are not prohibitive, the method offers a positive means of analysis."

In the 1950's, several attempts were made to perform surface analysis following bombardment of the surface with electrons [21-23]. However, because their number is small in comparison to the secondary and backscattered electrons, it was difficult to discriminate the Auger electrons from the general background. In 1967, Harris markedly improved the signal-to- noise ratio by associating phase-sensitive detection with an electrostatic sector velocity analyser [24]. Following a discussion with Harris, Peria realised that the standard LEED equipments could be used to perform AES work with minor electronic modifications [25]. The next significant technical improvment came in 1969 with the introduction by Palmberg et al. of the cylindrical mirror analyser [26]. Commercial instruments were soon available and Auger electron spectroscopy became the most commonly used experimental technique for the determination of surface chemical composition.

$\mathrm{X}$-ray electron spectroscopy has its origins in the discovery of the photoelectric effect in 1887. However, for much of this early period, it was used to probe the energy level structure of matter and could not compete in accuracy with X-ray absorption and X-ray emission spectroscopies. In the 1950s, several instruments were conceived by Steinhardt and Serfass to perform surface chemical analysis using XPS [27]. But the accurate measurement of atomic electron binding energies and of shifts in the position of photoelectron and Auger lines has only been made possible with the use of a high-resolution double focusing electron analyser as proposed by Siegbahn and coworkers during the period 1955-1970 [28]. The acronym ESCA (electron spectroscopy for chemical analysis) was given to the technique to point out that both photoelectrons and Auger electrons were observed in the "XPS" spectra. As for AES, commercial instruments in ultrahigh vacuum environment became available in the early 1970s, leading to an increasing use of XPS as a surface technique.

The sampling depth of any electron spectroscopy is determined by the inelastic scattering of the emitted electrons. For electrons in the energy range $30-2000 \mathrm{eV}$, the inelastic mean free path, which is inversely proportional to the inelastic scattering cross section, varies from about $1 \mathrm{~nm}$ to $5 \mathrm{~nm}$ for most materials [29, 30]. Because all materials (with the exception of hydrogen and helium) can give rise to Auger or photoelectrons in this energy range, both AES and XPS can achieve surface sensitivity and are still now dominating the field of surface science, especially for probing the electronic properties of surface and for determining the elementary composition. 
4.1 Electronic Properties of Surfaces. - The electronic properties of surfaces are often investigated by using Auger lineshapes in combined XPS and AES studies. The low energy Auger features typically observed in surface studies are often CVV transitions and it was early pointed out by Lander that they could yield information about band structure [21]. For an Auger transition which creates two final-state holes in the valence band, the Auger peak should be given by the difference in binding energy of the initial state and the self-convolution of the single-electron density of states. However, Powell has experimentally shown that the Auger-transition matrix element is energy dependent and that the correlation energy between the two holes cannot be neglected [31]. Cini [32] and Sawatzky [33] suggested that quasi-atomic Auger spectra, accompanied by broad band-like peaks at higher energies, arise when the correlation energy between two holes localised on atomic site is much greater than the single-electron bandwith of the material under study. When the Coulomb interaction is small, the Auger spectra should be band-like. The Cini-Sawatzky theory has proved extremely fruitful in the study of the local density of states of two components alloys, as shown by Weightman and co-workers [34].

However, the direct determination of the correlation energy between the two holes from the Auger spectrum following the Cini-Sawatzky theory looks hazardous, especially for incompletely filled bands materials. Some recent effort has been made by Treglia et al. [35] to generalize the Cini-Sawatzky model. The presence of inelastic electron scattering contributions and of satellites also add further complication. A new technique to identify core-line satellites has recently been proposed by Haak et al. [36], based on the measurement of an Auger spectrum in coincidence with the photoelectron peak corresponding to a specific core vacancy.

Even though there still remain several sources of uncertainties in the analysis of the Auger lineshapes, an increasing number of studies was dedicated to their use as a probe for surface electronic processes in the last two decades [for an overview, see 37] and they appear as a promising tool to gain further insight on the local electronic structures.

4.2 Surface Composition. - Because the energy of the Auger electron is low and characteristic of the emitting atom, Auger electron spectroscopy looks ideally suited for surface elemental analysis. However, some caution must be taken because electron-excited Auger electron spectroscopy (AES) can induce severe damage to some materials (like polymers) as well as charging effects to non-conducting specimen. These drawbacks can be circumvented by using XPS spectra, where Auger peaks are also present. Crucial to the determination of surface composition is the quantification of AES and XPS. In spite of all the efforts devoted to this problem, there is, so far, no unified methodology and quantification is usually derived either from first principle calculations, from data bases or from standards. The possible systematic errors associated with specimen, instruments or methodology have been recently reviewed [38].

As soon as electron-excited Auger electron spectroscopy (AES) was associated with LEED studies, as suggested by Weber and Peria [25], it was realised that many "clean" metallic surfaces were in fact covered by a monolayer of segregated impurities. This was indeed the first direct observation of the surface segregation effect theoretically predicted by Gibbs in 1875 [39]. Since that period and because of its unique high data acquisition rate capabilities, AES has been predominantly utilised to carry out segregation experiments.

It is often desirable to obtain the variation of chemical composition in the direction perpendicular to the surface. Such an in-depth distribution analysis is usually achieved by using AES or XPS in conjunction with ion sputtering to erode the surface specimen. The most difficult task in such an experiment is to convert the sputtering profile experimentally monitored (i.e. Auger signal as a function of sputtering time) in terms of original concentration profile. This requires a quantification of the signal intensity and the depth scale as well as a precise knowledge of the depth resolution function, as discussed by Hofmann [40]. 
Because most of the practical samples are also laterally inhomogeneous, the ability to provide a two-dimensional map of the chemical composition of the surface is often considered as a major advantage of AES. This capability was first demonstrated by McDonald in 1970 [41] and the first commercial scanning Auger microscope became available in 1973. Elementary surface mapping can also be achieved using photon excitation, but electron incident beams, which can be easily focused and rastered across the surface, still lead to a better lateral resolution. The most salient features of SAM are discussed in the companion paper by Prutton.

AES and XPS are now routinely used in a large number of applications, like microelectronics, metallurgy, heterogeneous catalysis, corrosion science, adhesion science, polymer technology... Detailed information on these applications can be found in a recent book [42].

\section{Epilogue}

It was more than 30 years before technological progress changed Pierre Auger's discovery from a laboratory curiosity into a powerful means of studying atoms and solid surfaces. Even though thère still remains a lot to be done, especially from a theoretical point of view, Auger electron spectroscopy is now playing a prominent role in the understanding of the basis physics as well as in the development of many advanced materials.

Fortunately, Pierre Auger lived long enough to see the formidable scientific and technological impact of his first discovery. In fact, the activities of his whole life can be characterised by a single word: novelty. The discovery of radiationless transitions was followed by the first experimental observation of spallation in the 1930s, and of the giant cosmic ray showers in the 1940s. After the war, he started a national and international civil service career, which lead him to promote the establishment of organisations like the french Atomic Energy Commission, the European Nuclear Research Center, the European Space Agency (previouly ESRO)...

Pierre Auger died in Paris, December 24th 1993.

\section{References}

[1] Auger P., C.R. Acad. Sci. Paris 177 (1923) 169.

[2] Wilson C.T.R., Proc. Roy. Soc. LXXXVII (1912).

[3] Ellis C.D., Proc. Roy. Soc. 99 (1921) 261.

[4] Meitner L., Z. Phys . 17 (1923) 54.

[5] Auger P., C.R. Acad. Sci. Paris 180 (1925) 65.

[6] Auger P., Thèse présentée à la Faculté des Sciences de Paris, le 26 juin 1926, devant la Commission d'Examen composée de Mme Marie Curie, présidente, MM Georges Urbain et Jean Perrin, examinateurs, published in Ann. Phys. 6 (1926) 183.

[7] Holweck F., Ann. Phys. 9ème série XVII 5 (1922).

[8] Wentzel G., Z. Phys. 43 (1927) 524.

[9] Coster D. and R. de Kronig L., Physica 2 (1935) 13.

[10] Howarth L.J., Phys. Rev. 48 (1935) 88.

[11] Kiang, Ma and Wu, Phys. Rev. 50 (1936) 673.

[12] Massey H.S.W. and Burhop E.H.S., Electronic and Ionic Impact Phenomena (Oxford, 1952). 
[13] Briant J-P., Le Vide, Les Couches Minces 271 (1994) 127.

[14] Bloch F., Phys. Rev. 48 (1935) 187.

[15] see Åberg T., Phys. Rev. A 4 (1971) 1735 and references therein.

[16] Meyer F.W., Griffin D.C., Havener C.C., Huq M.S., Phaneuf R.A., Swenson J.K. and Stolterfoht N., Phys. Rev. Lett. 60 (1988) 1821.

[17] Briand J.-P., de Billy L., Charles P., Essabaa S., Briand P., Geller R., Desclaux J-P., Bliman S. and Ristori C., Phys. Rev. Lett. 65 (1990) 159.

[18] Schuch R., Schneider D., Knapp D.A., DeWitt D., McDonald J., Chen M.H., Clark M.W. and Marrs R.E., Phys. Rev. Lett. 70 (1993) 1073.

[19] Massey H.S.W. and Bates D.R., Rep. Prog. Phys. 9 (1942) 62.

[20] Briand J-P., Charles P., Arianer J., Laurent H., Goldstein C., Dubau J., Loulergue M. and Bely-Dubau F., Phys. Rev. Lett 52 (1984) 617.

[21] Lander J.J., Phys. Rev. 91 (1953) 1382.

[22] Harrower G.A., Phys. Rev. 102 (1956) 340.

[23] Powell C.J., Robins J.L. and Swan J.B., Phys. Rev. 110 (1958) 657.

[24] Harris L.A., J. Appl. Phys. 39 (1968) 1419 and 1428.

[25] Weber R.E. and Peria W.T., J. Appl. Phys. 38 (1967) 4355.

[26] Palmberg P.W., Bohm G.K. and Tracy J.C., Appl. Phys. Lett. 15 (1969) 254.

[27] Steinhardt R.G. and Serfass E.J., Anal. Chem. 25 (1953) 697.

[28] Siegbahn K., Nordling C.N., Fahlman A., Nordberg R., Hamrin K., Hedman J., Johansson G., Benmark T., Karlsson S.E., Lindgren I. and Lindberg B. in ESCA: Atomic Molecular and Solid State Structure Studied by Means of Electron Spectroscopy, Almquist and Wiksells (Uppsala, 1967).

[29] Seah M.P. and Dench W.A., Surf. Interface Anal. 1 (1979) 2.

[30] Tanuma S., Powell C.J. and Penn D.R., Surf. Interface Anal. 17 (1991) 911; 20 (1993) 77.

[31] Powell C.J., Phys. Rev. Lett. 30 (1973) 1179.

[32] Cini M., Solid State Commun. 20 (1976) 605.

[33] Sawatzky G.A., Phys. Rev. Lett. 39 (1977) 504.

[34] Weightman P., Rep. Prog. Phys. 45 (1982) 753.

[35] Tréglia G., Desjonquères M-C., Ducastelle F. and Spanjaard D., J. Phys. C: Solid State Phys. 14 (1981) 4347.

[36] Haak H.W., Sawatzky G. and Thomas T.D., Phys. Rev. Lett. 41 (1978) 1825.

[37] Powell C.J., Le Vide, Les Couches Minces 271 (1994) 127.

[38] Powell C.J. and Seah M.P., J. Vac. Sci. Technol. A8 (1990) 735.

[39] Gibbs J.W., Trans. Conn. Acad. Sci. 3 (1875) 108.

[40] Hofmann S., in Practical Surface Analysis 1, D. Briggs and M.P. Seah Eds. (John Wiley and Sons Ltd, Chichester, 1990).

[41] McDonald N.C., Appl. Phys. Lett. 16 (1970) 76.

[42] D. Briggs and M.P. Seah, Practical Surface Analysis 1, Auger and X-ray Photoelectron Spectroscopy (John Wiley and Sons Ltd, Chichester, 1990). 